\title{
Representation of AC hysteretic characteristics of silicon steel sheet using simple excess eddy-current loss approximation
}

\author{
AUTHOR(S): \\ Matsuo, T; Terada, Y; Shimasaki, M
}

\section{CITATION:}

Matsuo, T ...[et al]. Representation of AC hysteretic characteristics of silicon steel sheet using simple excess eddy-current loss approximation. IEEE TRANSACTIONS ON MAGNETICS 2005, 41(5): 1544-1547

\section{ISSUE DATE:}

2005-05

URL:

http://hdl.handle.net/2433/40002

\section{RIGHT:}

(c)2005 IEEE. Personal use of this material is permitted. However, permission to reprint/republish this material for advertising or promotional purposes or for creating new collective works for resale or redistribution to servers or lists, or to reuse any copyrighted component of this work in other works must be obtained from the IEEE. 


\title{
Representation of AC Hysteretic Characteristics of Silicon Steel Sheet Using Simple Excess Eddy-Current Loss Approximation
}

\author{
Tetsuji Matsuo, Member, IEEE, Yasushi Terada, and Masaaki Shimasaki, Member, IEEE \\ Department of Electrical Engineering, Graduate School of Engineering, Kyoto University, Kyoto 615-8510, Japan
}

\begin{abstract}
A stop model is combined with a one-dimensional finite-difference method or a homogenization method to represent ac hysteretic characteristics of a silicon steel sheet. Eddy-current analysis without excess eddy-current loss fails to give an accurate eddy-current field. Representation of ac $B-H$ loops is improved by increased conductivity that is obtained from an excess loss evaluation by the Pry and Bean model.
\end{abstract}

Index Terms-AC hysteresis, anomaly factor, excess eddy-current loss, homogenization, Pry and Bean model, stop model.

\section{INTRODUCTION}

G RAIN-ORIENTED silicon steel sheets are widely used as a core material, not only for transformers, but also for segmented core motors. The grain-oriented steel sheets have a large excess (or anomaly) eddy-current loss [1] because of their relatively large magnetic domain size compared with nonoriented steel sheets [2]. An anomaly factor [2] is often used to evaluate the excess eddy-current loss, which does not require an accurate $B-H$ field distribution inside the steel sheet.

Recent development of computer technology enables us to simulate detailed $B-H$ fields in electrical machines. However, accurate computation of the $B-H$ field is not yet easy because of complex magnetic properties of the steel sheet including the excess eddy-current loss, hysteresis [1], and a vector property [3].

On the other hand, several precise dc hysteresis models have been developed, such as play and stop models [4], [5]. Those models are sufficiently efficient to be applied to magnetic field analyses. For example, a stop model having an input-dependent shape function [6], [7] has been proposed to accurately represent dc hysteretic characteristics of silicon steel sheets.

This study combines the stop model with a one-dimensional (1-D) finite-difference method or a homogenization method to represent ac hysteretic characteristics of a grain-oriented silicon steel sheet. A homogenized model is derived from the Pry and Bean model [8], where the effect of excess eddy-current loss is evaluated simply by an anomaly factor. This simple loss evaluation is also applied to the 1-D finite-difference analysis.

\section{EDDy-CuRRent ANALysis Without ExCESS LosS}

This study analyzes an eddy-current field in a grain-oriented silicon steel sheet (JIS: 30P105). This steel sheet has electric conductivity $\sigma$ of $2 \times 10^{6} \mathrm{~S} / \mathrm{m}$.

One-dimensional magnetic properties of the silicon steel sheet are measured using a single sheet tester [9] that applies the magnetic flux sinusoidally.
One-dimensional eddy-current fields are described by

$$
\frac{\partial^{2} H}{\partial y^{2}}=\sigma \frac{\partial B}{\partial t}
$$

where the $y$ direction is perpendicular to the rolling and transverse directions of the silicon steel sheet.

The finite-difference method is used to simulate the eddycurrent field, where a stop model having the input-dependent shape function [6], [7] represents the hysteretic relation $H=$ $H(B)$. The backward Euler time-difference scheme leads to

$$
\frac{H\left(B_{i+1}^{n}\right)-2 H\left(B_{i}^{n}\right)+H\left(B_{i-1}^{n}\right)}{(\Delta y)^{2}}=\sigma \frac{B_{i}^{n}-B_{i}^{n-1}}{\Delta t}
$$

where $B_{i}^{n}=\left.B\right|_{y=i \Delta y, t=n \Delta t}$. The surface magnetic field is given by the measured data as the boundary condition. The computed $B$ is averaged along the $y$ direction for comparison with measured data.

Simulated ac $B-H$ loops along the rolling and transverse directions are plotted in Figs. 1 and 2, respectively. The exciting frequencies are set at 20 and $50 \mathrm{~Hz}$. Figs. 1 and 2 show that the simulated loops disagree with measured ones because this analysis neglects the excess eddy-current loss [1] that is attributable to the concentration of eddy currents around the magnetic domain walls.

\section{PRY AND BEAN MODEL}

Pry and Bean [8] have evaluated the excess eddy-current loss assuming a 1-D periodic magnetic domain structure. Fig. 3 illustrates this structure: $2 L$ is the average domain width, $x_{\mathrm{W}}$ is the wall displacement from the demagnetized position, and $d$ is the sheet thickness.

The electric current density $j=\left(j_{\mathrm{x}}, j_{\mathrm{y}}\right)$ satisfies the following equations in the analyzed region: $-L \leq x \leq L$ and $-d / 2 \leq y \leq d / 2$ 

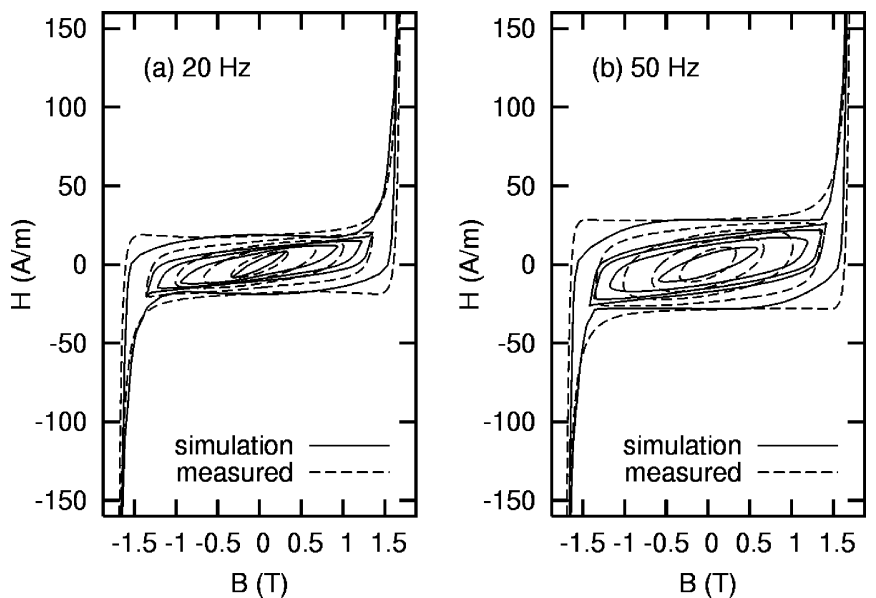

Fig. 1. AC $B-H$ loops along the rolling direction given by eddy-current analysis.
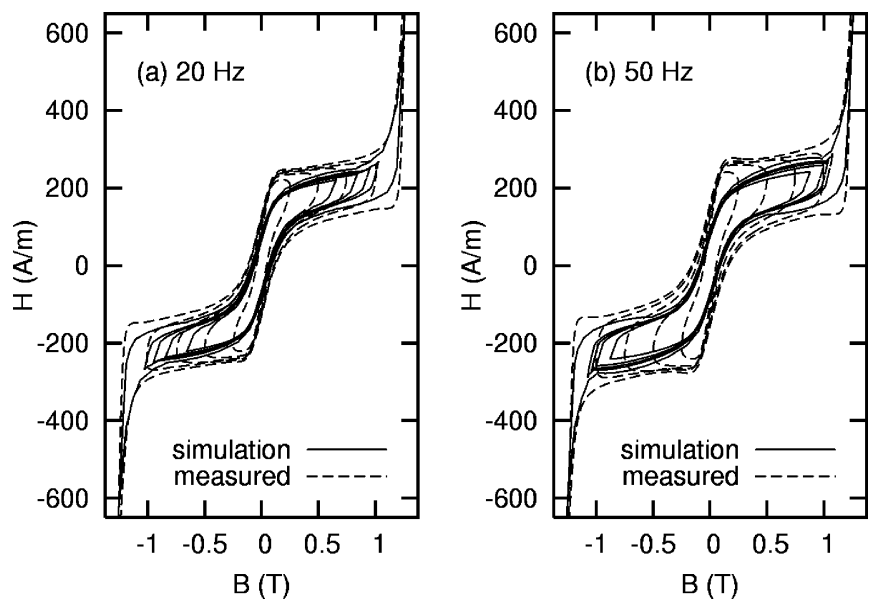

Fig. 2. AC $B-H$ loops along the transverse direction given by eddy-current analysis.

(a)

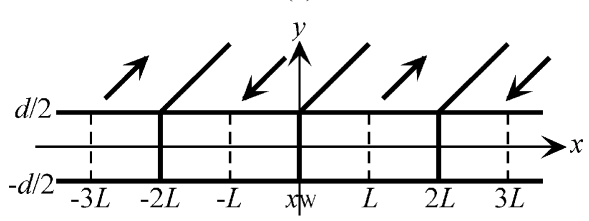

(b)

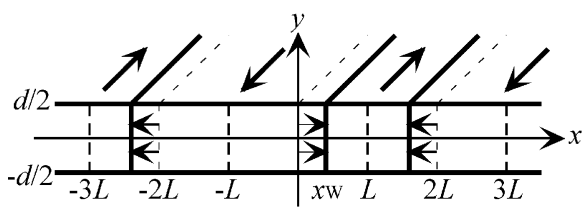

Fig. 3. One-dimensional periodic magnetic domain structure. (a) Demagnetized. (b) Magnetized.

The boundary condition is given as

$$
\begin{aligned}
& \left.j_{\mathrm{x}}\right|_{x=x \mathrm{w}+0}=\left.j_{\mathrm{x}}\right|_{x=x \mathrm{w}-0}, \\
& \left.j_{\mathrm{y}}\right|_{x=x \mathrm{w}+0}-\left.j_{\mathrm{y}}\right|_{x=x \mathrm{w}-0}=-2 \sigma M_{\mathrm{S}} v_{\mathrm{W}} \\
& j_{\mathrm{y}}=0(x= \pm L), \quad j_{\mathrm{y}}=0\left(y= \pm \frac{d}{2}\right) .
\end{aligned}
$$

where $M_{\mathrm{S}}$ is the saturation magnetization and $v_{\mathrm{W}}=\mathrm{d} x_{\mathrm{W}} / \mathrm{d} t$ is the wall velocity.

Thereby, the instantaneous power loss per unit volume $P(=$ $\left.\iint\left(j_{\mathrm{x}}^{2}+j_{\mathrm{y}}^{2}\right) / \sigma \mathrm{d} x \mathrm{~d} y / \iint \mathrm{d} x \mathrm{~d} y\right)$ becomes

$$
P=\frac{16 \sigma L d}{\pi^{3}}\left(\frac{\mathrm{d} B}{\mathrm{~d} t}\right)^{2} \sum_{n \text { odd }}^{\infty} \frac{\cosh \frac{n \pi\left(L+x_{\mathrm{W}}\right)}{d} \cosh \frac{n \pi\left(L-x_{\mathrm{W}}\right)}{d}}{n^{3} \sinh \frac{2 n \pi L}{d}}
$$

where $B=M_{\mathrm{S}} x_{\mathrm{W}} / L$ is the average magnetic flux density. When $L \ll d, P$ is reduced to the classical eddy-current loss $P_{\mathrm{C}}$ given by

$$
P_{\mathrm{C}}=\frac{\sigma d^{2}}{12}\left(\frac{\mathrm{d} B}{\mathrm{~d} t}\right)^{2} .
$$

On the other hand, when $B$ is small $\left(x_{\mathrm{W}} \ll L\right), P$ is approximated as

$$
P=\frac{8 \sigma L d}{\pi^{3}}\left(\frac{\mathrm{d} B}{\mathrm{~d} t}\right)^{2} \sum_{n \text { odd }}^{\infty} \frac{1}{n^{3}} \operatorname{coth} \frac{n \pi L}{d}=\sigma \frac{k_{E} d^{2}}{12}\left(\frac{\mathrm{d} B}{\mathrm{~d} t}\right)^{2}
$$

where $k_{\mathrm{E}}$ is the anomaly factor given by

$$
k_{\mathrm{E}}=\frac{P}{P_{\mathrm{C}}}=\frac{96 L}{\pi^{3} d} \sum_{n \text { odd }}^{\infty} \frac{1}{n^{3}} \operatorname{coth} \frac{n \pi L}{d} .
$$

For simplicity, this paper uses this factor to approximate $P$ as $P=k_{\mathrm{E}} P_{\mathrm{C}}$, independently of $B$.

\section{HOMOGENIZED MODEL}

By setting

$$
H_{\mathrm{E}}=k_{\mathrm{E}} \frac{\sigma d^{2}}{12} \frac{\mathrm{d} B}{\mathrm{~d} t}
$$

the power loss $P$ is factorized as

$$
P=H_{\mathrm{E}} \frac{\mathrm{d} B}{\mathrm{~d} t} .
$$

The field $H_{\mathrm{E}}$ can be regarded as an averaged magnetic field at the domain walls [1], which is required to move the domain walls against the counter force by the eddy current. Accordingly, the applied field $H_{\mathrm{a}}$ is decomposed into

$$
H_{\mathrm{a}}=H_{\mathrm{E}}+H_{\mathrm{DC}}
$$

where $H_{\mathrm{DC}}$ is a rate-independent field [1] that causes a dc hysteresis.

Consequently, a homogenized model including the excess eddy-current loss is given as

$$
H_{\mathrm{a}}(t)=H_{\mathrm{DC}}(B(t))+k_{\mathrm{E}} \frac{\sigma d^{2}}{12} \frac{\mathrm{d} B}{\mathrm{~d} t}
$$

where $H_{\mathrm{DC}}(B)$ represents the dc hysteresis property.

Several homogenized models having similar forms to (13) have already been presented in the literature. For example, homogenization without the excess loss $\left(k_{\mathrm{E}}=1\right)$ is given in [10]. A more mathematically general form than (13) is discussed in [11].

AC $B-H$ loops of the grain-oriented silicon steel sheet are simulated by the homogenized model (13), where the applied 

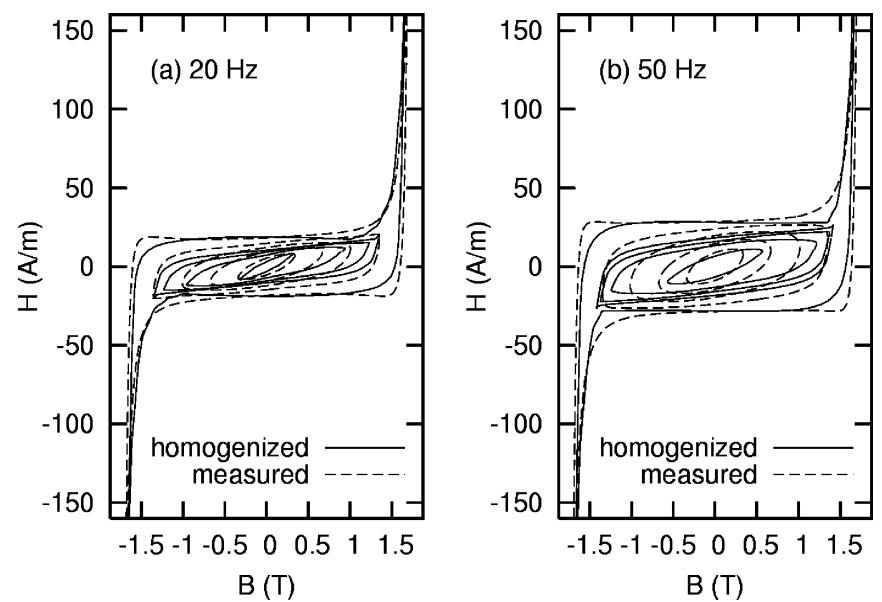

Fig. 4. AC $B-H$ loops along the rolling direction given by the homogenized model with $k_{\mathrm{E}}=1$.
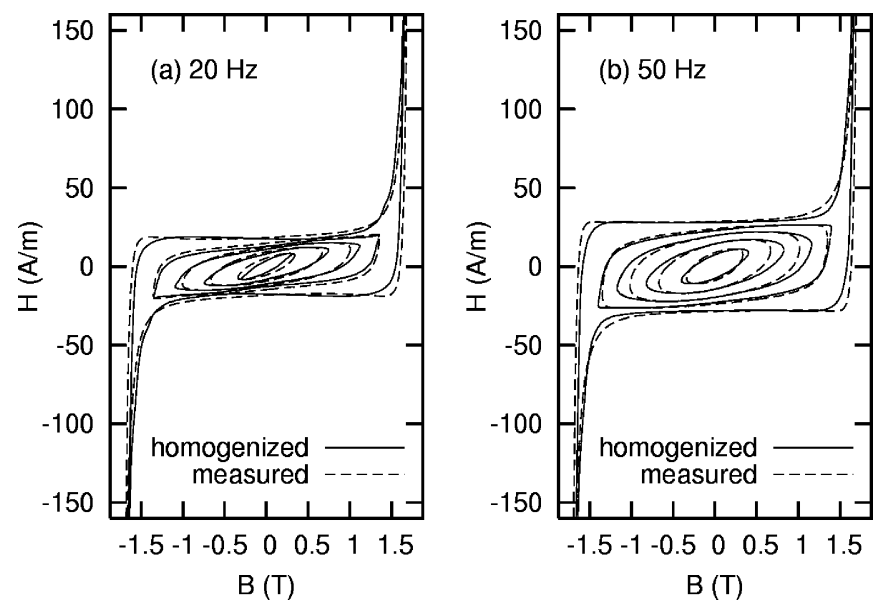

Fig. 5. AC $B-H$ loops along the rolling direction given by the homogenized model with $k_{\mathrm{E}}=2.5$.

field $H_{\mathrm{a}}(t)$ is given by the measured data for comparison with the finite-difference eddy-current analysis.

Figs. 4 and 5 show simulated $B-H$ loops along the rolling direction, where $k_{\mathrm{E}}=1$ and 2.5 are used for the homogenized model, respectively. The factor $k_{\mathrm{E}}=2.5$ corresponds to $2 L / d \approx 1.5$. Fig. 4 shows that homogenization without the excess eddy-current loss engenders a large discrepancy between the simulated and measured loops in the same way as the 1-D finite-difference analysis failed. Fig. 5 shows that the factor $k_{\mathrm{E}}=2.5$ improves the representation of ac $B-H$ loops effectively.

Figs. 6 and 7 show simulated ac $B-H$ loops along the transverse direction, where $k_{\mathrm{E}}=1$ and 10 (corresponding to $2 L / d \approx 6$ ) are used, respectively. Fig. 6 shows that the simulation without the excess eddy-current loss fails to yield accurate $B-H$ loops. The factor $k_{\mathrm{E}}=10$ improves the representation of ac $B-H$ loops. The anomaly factor required for the transverse direction is much larger than that for the rolling direction because of the large domain size. However, a large discrepancy remains between the simulated and measured loops mainly because the Pry and Bean model cannot describe the $90^{\circ}$ domain wall motion that dominates the magnetic property along the transverse direction.
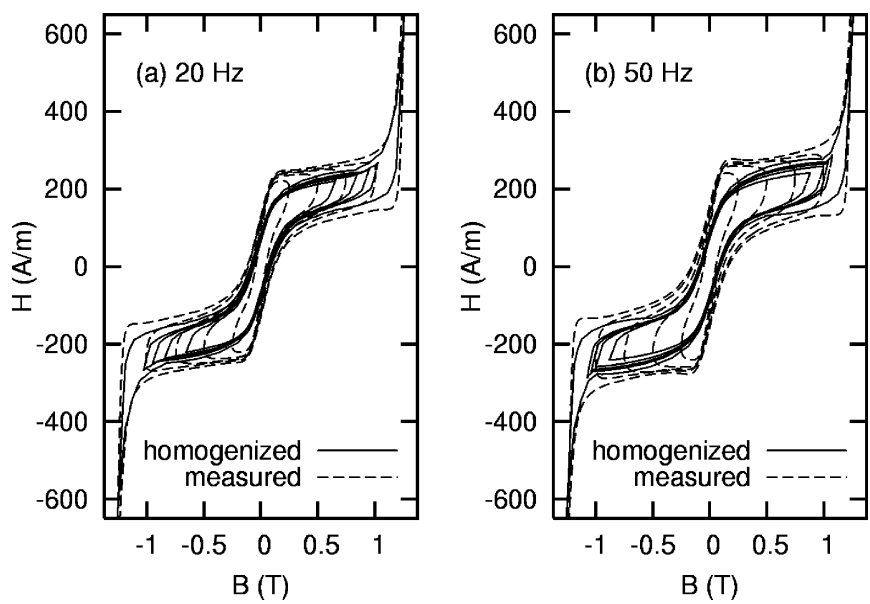

Fig. 6. $\mathrm{AC} B-H$ loops along the transverse direction given by the homogenized model with $k_{\mathrm{E}}=1$.
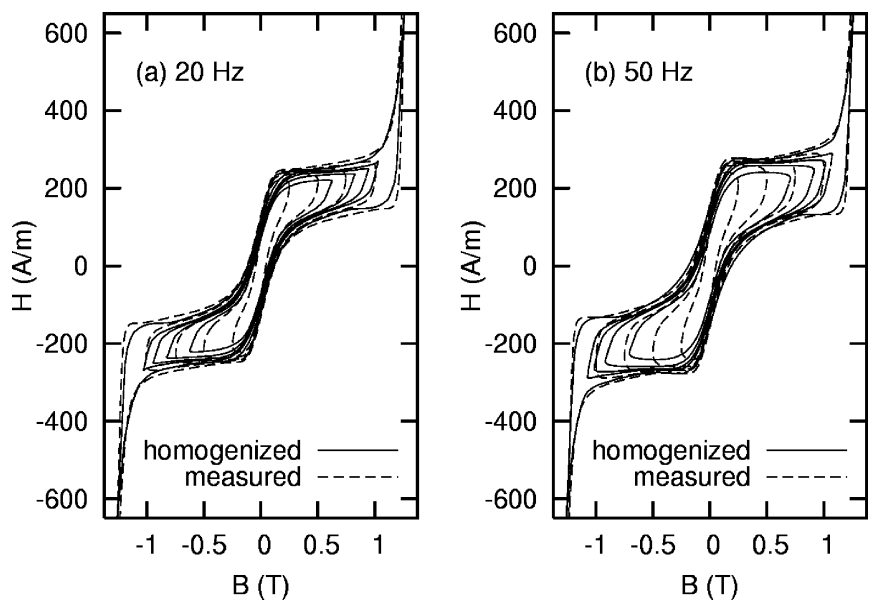

Fig. 7. AC $B-H$ loops along the transverse direction given by the homogenized model with $k_{\mathrm{E}}=10$.

\section{Finite-Difference ANALYsis InCLUding ExCESS Loss}

This paper simply multiplies electric conductivity by the factor $k_{\mathrm{E}}$ for the 1-D finite-difference analysis to approximate the effect of excess eddy-current loss in the same way as in the homogenized model

$$
\frac{\partial^{2} H}{\partial y^{2}}=\sigma^{\prime} \frac{\partial B}{\partial t}, \quad \sigma^{\prime}=k_{\mathrm{E}} \sigma
$$

Fig. 8 shows simulated $B-H$ loops along the rolling direction obtained by analysis using $k_{\mathrm{E}}=2.5$, where the increased conductivity improves the representation of the $B-H$ loops compared with Fig. 1. Table I lists the discrepancy (\%) between the simulated and measured power loss $W=\oint H \mathrm{~d} B$ of five $B-H$ loops at $50 \mathrm{~Hz}$, where $B_{\max }$ denotes the maximum magnetic field of each measured $B-H$ loop. Table I shows that the factor $k_{\mathrm{E}}=2.5$ greatly improves the power loss evaluation. However, more than $10 \%$ of the discrepancy remains between the computed and measured losses because the excess loss evaluation by the constant anomaly factor is too simple to represent an accurate eddy-current field. Table I and the comparison between Figs. 5 and 8 show that the finite-difference model does 

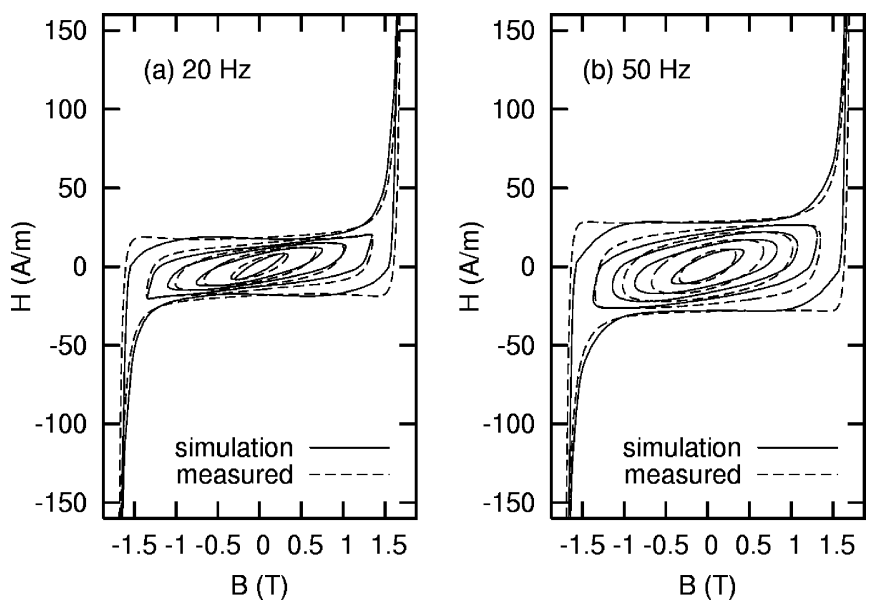

Fig. 8. AC $B-H$ loops along the rolling direction given by eddy-current analysis with $k_{\mathrm{E}}=2.5$.

TABLE I

DisCREPANCY (\%) BeTwEEn THE SimUlated AND MEASURED POWER LoSS

\begin{tabular}{c||c|c|c|c}
\hline \multicolumn{1}{c||}{$B \operatorname{cmax}(\mathrm{T})$} & \multicolumn{2}{c|}{ finite difference } & \multicolumn{2}{c}{ homogenization } \\
\cline { 2 - 5 } & $k_{\mathrm{E}}=1$ & $k_{\mathrm{E}}=2.5$ & $k_{\mathrm{E}}=1$ & $k_{\mathrm{E}}=2.5$ \\
\hline 0.34 & +22.1 & +0.6 & +18.4 & -3.8 \\
\hline 0.67 & +58.6 & +17.7 & +75.5 & +17.8 \\
\hline 1.01 & +10.4 & -2.0 & +17.0 & +11.3 \\
\hline 1.34 & -24.2 & -14.5 & -20.1 & -2.2 \\
\hline 1.68 & -19.1 & -8.6 & -14.3 & -8.9 \\
\hline
\end{tabular}
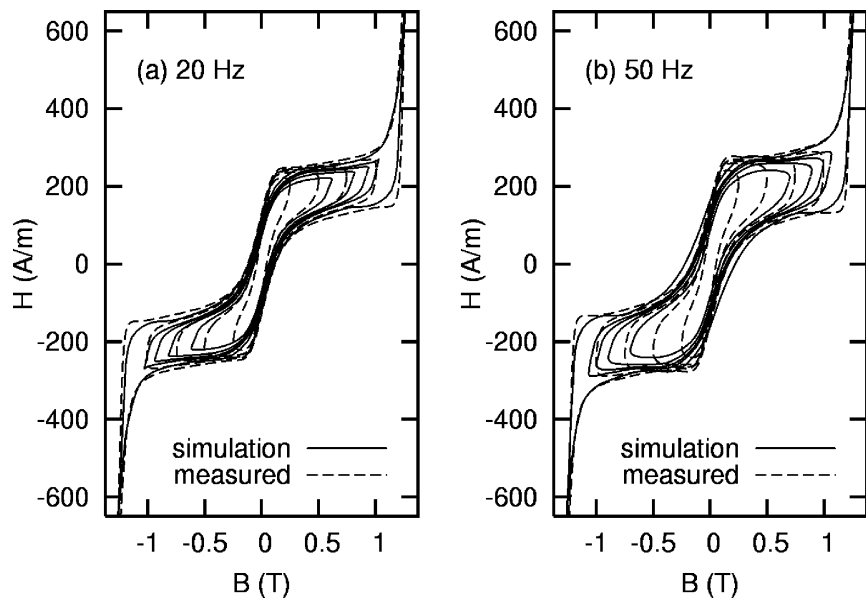

Fig. 9. AC $B-H$ loops along the transverse direction given by eddy-current analysis with $k_{\mathrm{E}}=10$.

not greatly improve the representation accuracy of the eddy-current field compared with the homogenization model. There is little improvement because the excitation frequency is too low for the skin effect to become sufficiently large.

Fig. 9 shows $B-H$ loops along the transverse direction simulated with $k_{\mathrm{E}}=10$, where the representation of the $B-H$ loops is improved compared with Fig. 2. However, a large discrepancy remains between the simulated and measured small $B-H$ loops. This fact means that the excess loss approximation by the constant anomaly factor is not valid for the transverse direction because of the $90^{\circ}$ domain wall motion that the Pry and Bean model cannot describe.

\section{CONCLUSION}

This paper combines a 1-D finite-difference method or a homogenization method with a stop model to describe ac hysteretic properties of a grain-oriented silicon steel sheet. A homogenized model is derived from the Pry and Bean model, where the excess eddy-current loss is evaluated by an anomaly factor. An increased electric conductivity by the anomaly factor improves the representation of ac $B-H$ loops by the 1-D finite-difference method as effectively as by the homogenization method.

\section{ACKNOWLEDGMENT}

This work was supported in part by the Kansai Research Foundation for Technology Promotion.

\section{REFERENCES}

[1] G. Bertotti, Hysteresis in Magnetism. San Diego, CA: Academic, 1998.

[2] C. Kaido and T. Wakisaka, "Effect of material parameters on iron losses in nonoriented electrical steel sheets," Trans. Inst. Elect. Eng. Jpn. A, vol. 117, pp. 685-690, Jul. 1997.

[3] M. Enokizono, T. Todaka, S. Kanao, and J. Sievert, "Two-dimensional magnetic properties of silicon steel sheet subjected to a rotating field," IEEE Trans. Magn., vol. 29, no. 6, pp. 3550-3552, Nov. 1993.

[4] M. A. Krasnosel'skii and A. V. Pokrovskii, Systems With Hysteresis. Berlin, Germany: Springer-Verlag, 1989.

[5] S. Bobbio, G. Miano, C. Serpico, and C. Visone, "Models of magnetic hysteresis based on play and stop hysterons," IEEE Trans. Magn., vol. 33, no. 6, pp. 4417-4426, Nov. 1997.

[6] T. Matsuo, Y. Terada, and M. Shimasaki, "Stop model with input-dependent shape function and its identification methods," IEEE Trans. Magn., vol. 40, no. 4, pp. 1776-1783, Jul. 2004.

[7] T. Matsuo and M. Shimasaki, "Representation theorems for stop and play models with input-dependent shape functions," IEEE Trans. Magn., to be published.

[8] R. H. Pry and C. P. Bean, "Calculation of the energy loss in magnetic sheet materials using a domain model," J. Appl. Phys., vol. 29, pp. 532-533, Mar. 1958.

[9] T. Nakase, M. Nakano, K. Fujiwara, and N. Takahashi, "Method of digital waveform control for measuring magnetic properties by means of a single sheet tester," Trans. Inst. Elect. Eng. Jpn. A, vol. 119, pp. 1019-1025, Jul. 1999.

[10] A. J. Bergqvist and S. G. Engdahl, "A homogenization procedure of field quantities in laminated electric steel," IEEE Trans. Magn., vol. 37, no. 5, pp. 3329-3331, Sep. 2001.

[11] S. E. Zirka, Y. I. Moroz, P. Marketos, and A. J. Moses, "Dynamic hysteresis modeling," Physica B, vol. 343, pp. 90-95, 2004.

Manuscript received June 8, 2004. 